\title{
Border Definition
}

National Cancer Institute

\section{Source}

National Cancer Institute. Border Definition. NCI Thesaurus. Code C69220.

A description of the boundary findings or features of a sample. 